\title{
Efecto de un programa de actividades deportivas extraescolares en jóvenes chilenos Effect of an extracurricular sport activity program on young Chileans
}

*Cecilia Bahamonde, *Clemente Carmona, *Jabiela Albornoz, **Raquel Hernández-Garcia, ***Gema Torres-Luque

*Universidad Metropolitana de Ciencias de la Educación (Chile), **Universidad de Murcia (España), ***Universidad de Jaén (España)

Resumen. El objetivo de este trabajo fue determinar la influencia de un programa de actividad física extraescolar convencional de 12 semanas de

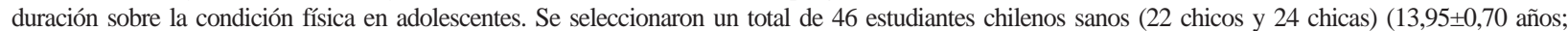

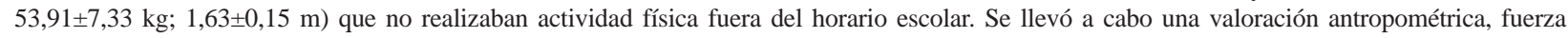
isométrica manual, salto horizontal, flexibilidad isquiosural, test 4x10m, test de CAFRA y test de balance «Y», antes y después de un programa de actividad física de 12 semanas de duración, 3 veces por semana y 60 minutos por sesión. Los resultados muestran que existen mejoras a nivel cardiovascular, salto de longitud y equilibrio dinámico; obteniendo valores más bajos en flexibilidad. A su vez, el género masculino mejora estadísticamente en salto horizontal y equilibrio dinámico; mientras que el género femenino lo hace, además, a nivel cardiovascular. Se pone de manifiesto la necesidad de evaluar programas de actividad física extraescolares convencionales en población joven y sana.

Palabras clave: adolescentes, condición física, entrenamiento, actividad física.

Abstract. The aim of this study was to determine the influence of a 12-week conventional physical activity program on the physical fitness of a group of adolescents. A total of 46 healthy Chilean students (22 boys and 24 girls) (13.95 \pm 0.70 years, $53.91 \pm 7.33 \mathrm{~kg}, 1.63 \pm 0.15 \mathrm{~m}$ ) who did not use to perform physical activity out of the school schedule were selected. Anthropometric assessment, hand grip, horizontal jump, hamstring flexibility, 4x10m test, CAFRA test and «Y» balance test were performed before and after a 12-week physical activity program characterized by 3 60-minute sessions per week. The results showed improvements in cardiovascular level, horizontal jump, and dynamic balance; however, lower values were found for flexibility. Boys improved statistically in horizontal jump and dynamic equilibrium; whereas girls gained also at the cardiovascular level. The need to evaluate conventional extracurricular physical activity programs in young and healthy populations is evident.

Key word: adolescent, physical condition, training, physical activity.

\section{Introducción}

Actualmente, es indiscutible el interés mundial que existe sobre la inactividad física. La organización mundial de la salud (WHO, 2009) estimó en el año 2009, que aproximadamente 155 millones de niños en edad escolar padecían de obesidad, estando uno de cada diez niños con un diagnóstico de sobrepeso u obesidad (Wang \& Lim, 2012). Este problema se hace extensible a la población chilena, donde la inactividad física se ha relacionado directamente con problemas de obesidad (Burrows, Diaz, Sciaraffia, Gattas, Montoya \& Lera, 2008; Godard, Román, Rodríguez, Leyton \& Salazar, 2012). Este hecho, está movilizando al mundo en busca de estrategias integrales y de promoción de estilos de vida saludables (Waters et al., 2011).

Acciones como leer libros o estar sentados frente al televisor tienen un efecto directo sobre riesgos cardiovasculares (Martínez-Gomez et al., 2010) o problemas de sueño (Ortega et al., 2008). En contrapartida, existen estudios que muestran la necesidad de la actividad física como herramienta para reducir perfiles lipídicos (Garcia-Hermoso, CarmonaLópez, Saavedra \& Escalante, 2014), adiposidad o riesgo cardiometabólico (Martinez-Vizcaino et al., 2014).

La etapa escolar, se ha destacado como un lugar idóneo para la adquisición y adherencia a la actividad física, fundamentalmente en las clases de educación física, el recreo y las actividades extraescolares (Calahorro-Cañada, Torres-Luque, López-Fernandez \& Carnero, 2015; Brazendale et al., 2017). Se ha mostrado la importancia de la actividad física recreativa no competitiva para el aprendizaje del placer del movimiento, independientemente de la habilidad del sujeto o el género (Martinez-Vizcaino et al., 2014). En concreto, la realización de actividades físicas extraescolares está asociada a un mayor rendimiento académico (Cladellas, Muntada, Badia \& Gotzens, 2013; Cladellas, Muntada, Gotzens, Badia \& Dezcallar, 2015); mayor nivel de atención y nivel educativo (Mahoney, Cairns \& Farmer, 2003) o incremento de la autoestima (Bailey, 2006).

A su vez, se ha demostrado como programas de entrenamiento con una duración de entre 8 y 12 semanas tiene una influencia en las capacidades físicas como la flexibilidad, capacidad de fuerza o resistencia (Venturelli, Trentin \& Bucci, 2007; Carrasco-Fernández, Lara-Sánchez

Fecha recepción: 15-01-18. Fecha de aceptación: 19-10-18 Gema Torres-Luque

gtluque@ujaen.es
\& Torres-Luque, 2014); fundamentalmente en poblaciones deportistas. Asu vez, se ha observado en poblaciones adolescentes la existencia de diferencias en la condición física respecto al género, donde los chicos obtienen mejores valores (Valdes \& Yanci, 2016), exceptuando generalmente la flexibilidad, donde existe una tendencia inversa (Casterad, \& Ostariz, 2005)

A pesar de existir información descriptiva sobre condición física, la importancia de valorar el nivel de actividad física o evaluar programas de intervención en población deportista, existe mucha menos información sobre el efecto desde el punto de vista físico-funcional de los programas de actividades extraescolares sin un fin de rendimiento, sino desde un punto de vista recreativo-saludable. Se considera necesaria la evaluación de programas específicos de intervención (Molinero et al., 2010).

Por lo tanto, el objetivo de este estudio es valorar los efectos de un programa de 12 semanas de duración de actividades extraescolares sobre la condición física en sujetos chilenos de entre 13 y 14 años; y observar las posibles diferencias en relación al género.

\section{Material y método}

\section{Muestra}

Participaron un total de 46 sujetos sanos chilenos de entre 13 y 14

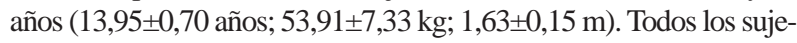
tos fueron clasificados según previos estudios (Villa Vicente et al., 2007; Torres-Luque, Carpio, Lara-Sánchez \& Zagalaz-Sánchez, 2014) como alumnos que sólo realizaban un total de 3 horas semana de actividad física, incluyendo las sesiones de educación física en su centro escolar.

La muestra fue seleccionada de forma intencional, en un centro educativo privado de Santiago de Chile, donde los criterios de inclusión fueron: tener entre 13 y 14 años, no tener ninguna enfermedad que impidiese la práctica de actividad física, tener un índice de masa corporal clasificada como normopeso y no faltar a más de dos sesiones del programa de actividad física. Se invitó a un total de 60 estudiantes que cumplían con los criterios de inclusión, treinta chicos y treinta chicas. En el grupo de chicos, se excluyeron 8 sujetos por no cumplir la asistencia mínima solicitada; mientras que en grupo de chicas se excluyeron a 6, conformando una muestra total de 46 sujetos. Tanto el centro educativo, como los padres, madres y/o tutores fueron informados de los objetivos del estudio, presentando un consentimiento informado por escrito para poder participar en el mismo. El estudio fue realizado de acuerdo con los procedimientos establecidos en la Declaración de Helsinki de la Asociación Médica Mundial (2008) sobre principios éticos para 
las investigaciones médicas en seres humanos.

\section{Procedimiento}

Se llevó a cabo una evaluación inicial (pre-test), un programa de actividad física extraescolar de 12 semanas de duración y una evaluación final (post-test). Las pruebas seleccionadas están prescritas para edades escolares incluyéndose en la batería APLHA(Ruiz et al., 2011); en vez del test de Cafra que es una prueba específica del Ministerio de Educación Chileno (MINEDUC, 2011); y la prueba de equilibrio dinámico (Pislky, Gorman, Butler, Kiesel, Underwood \& Elkins, 2009). Tanto el pre-test como el post-test se llevó a cabo en las mismas condiciones, con el mismo protocolo y a la misma hora del día en las dos ocasiones. El orden de las pruebas se realizó según se describen a continuación. Asu vez, todas las pruebas se evaluaron por el mismo investigador, el cual, estaba previamente familiarizado con este tipo de pruebas. Además, todos los sujetos estaban familiarizados con los test, ya que son empleados en las clases de educación física del centro escolar. En la Figura 1, se muestra la temporalización del estudio. posición se le solicitó que realizara una flexión máxima del tronco manteniendo las rodillas y los brazos extendidos. Las palmas de las manos, una al lado de la otra, se deslizaron sobre el cajón hasta alcanzar la máxima distancia posible. Los participantes realizaron dos intentos anotando el mayor de los dos en $\mathrm{cm}$.

Con una diferencia de 48h, en las mismas instalaciones, se llevaron a cabo los test que faltaban, las cuales se realizaron en el orden en que vienen descritas. Para ello, se realizó un calentamiento similar al que realizó en el día previo.

Test 4x10m: Test de correr y girar a la máxima velocidad. Dos líneas paralelas se dibujaron en el suelo a 10 metros de distancia. En la línea de salida hay una esponja (B) y en la línea opuesta había dos esponjas (A, C). Cuando se indicó la salida, el niño/a (sin esponja) corrió lo más rápido posible a la otra línea y volvía a la línea de salida con la esponja (A), cruzando ambas líneas con los dos pies. La esponja (A) se cambió por la esponja B en la línea de salida. Luego, fue corriendo lo más rápido posible a la línea opuesta, cambió la esponja B por la esponja C y volvió corriendo a la línea de salida. Se realizará dos veces y se anotará el mejor tiempo obtenido.
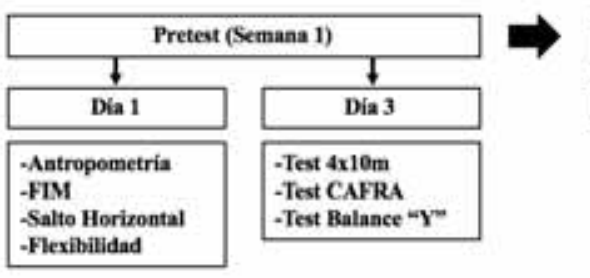

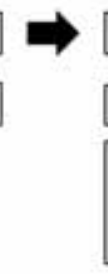

Figura 1. Cronograma

Valoración antropométrica: se determinó mediante la medición de la masa corporal (kg), la talla (cm) y el perímetro de la cintura (cm) en un aula habilitada para tal efecto. La medición del peso se realizó descalzos y con ropa interior o traje de baño, los participantes se colocaron de pie sobre la báscula eléctrica modelo SECA(SECALTD., Germany). Para la talla, se midieron a los participantes descalzos, de pie, con los talones, glúteos y espalda en contacto con la pared, con un tallímetro modelo SECA(SECALTD., Germany). Finalmente, el perímetro de la cintura se midió en el nivel más estrecho, entre el borde del costal inferior (décima costilla) y la cresta iliìaca, al final de una espiracioìn normal y sin que la cinta presionase la piel. Se empleó la cinta métrica inextensible milimetrada de fibra de vidrio Holtain.

Posteriormente, los ejecutantes realizaron un calentamiento de componente aeróbico (5 min) junto a movilidad articular de extremidades superiores (3 min), finalizándolo con unos ejercicios de estiramientos activos dinámicos de (3 min). Una vez completado el calentamiento estandarizado se comenzaron las siguientes pruebas.

Fuerza isométrica manual: cada alumno se situó de pie, erguido, con las piernas ligeramente separadas y los brazos extendidos verticalmente a lo largo del tronco, pero sin tocar ninguna parte de éste. Con esta posición el participante presionó lo máximo posible el Dinamómetro Manual Test 320, flexionando los dedos de la mano. En el momento en que consiguió su grado máximo de flexión se registróla marca en kilogramos. Se admitieron dos intentos con cada mano, con un minuto de recuperación, donde se realizaba primero con la mano derecha y después con la izquierda. Se registró la medición más elevada en cada mano en kilogramos.

Salto horizontal: consistió en saltar con los pies juntos desde una línea, sin carrera previa en una zona horizontal y antideslizante. El sujeto podía ayudarse del salto con movimiento de brazos. Se midió la distancia entre el talón del pie más retrasado y la línea de salida. Se realizan dos intentos y se anota el de mayor valor.

Flexibilidad Isquiosural: los alumnos se situaron en sedestación, con las rodillas extendidas y los pies separados a la anchura de sus caderas, con tobillos en $90^{\circ}$ de flexión. Las plantas de los pies se colocaron perpendiculares al suelo, en contacto con el cajón de medición (marca Eveque) y las puntas de los pies mirando hacia arriba. En esta
Test de CAFRA: es una prueba estimativa de la capacidad aeróbica en condiciones sub-máxima a través del consumo de oxígeno por unidad de peso corporal correspondiente a la velocidad aplicada, al peso corporal y a la frecuencia cardíaca alcanzada al final del test (MINEDUC, 2011). El sujeto caminó (ni trotó ni corrió) por un pentágono de 10 metros cada lado, manteniendo una velocidad constante de $6 \mathrm{~km} / \mathrm{h}$, $7 \mathrm{~km} / \mathrm{h}$ y $8 \mathrm{~km} / \mathrm{h}$ durante 3 minutos para cada carga, al ritmo de un estímulo sonoro. Al término de la prueba, se controló la frecuencia cardiaca por medio de un pulsometro (Polar F4, Finland). Cada una de las pruebas fue separada por un minuto de descanso. Posteriormente, y de forma indirecta se obtiene el consumo máximo de oxígeno $\left(\mathrm{VO}_{2}\right.$ máx.= ((20·(220-edad)) /FC del test); MINEDUC, 2011).

Test de Balance «Y»(TBY): se llevó a cabo una prueba para evaluar el equilibrio dinámico de los sujetos. Para ello se empleó el protocolo estandarizado de Y Balance Test (Plisky et al., 2009). Los sujetos se colocaban sobre un solo pie con su calzado deportivo en una placa central donde se solicitaba que extendiese lo máximo posible la pierna libre hacia delante, desplazando un dispositivo que mide la distancia en centímetros. Posteriormente se solicitaba el mismo procedimiento hacia el lado un lado y detrás del mismo lado; y, por último, el mismo procedimiento hacia el lado contrario conformando una figura en « $Y$ », Todos los sujetos estaban familiarizados con el test; realizándolo tres veces con cada pierna seleccionando el valor máximo.

Programa de actividad física extraescolar

Se llevó a cabo un programa de actividad física extraescolar de 12 semanas de duración, tres veces por semana, con una duración de cada sesión de 60 minutos. Las actividades fueron desarrolladas en grupos de 10-12 personas, en un centro habilitado para tal efecto, donde el contenido era fundamentalmente la ejecución de deportes de carácter individual (taekwondo, atletismo, patinaje) o colectivo (hockey, balonmano, baloncesto). Todas las sesiones tuvieron la misma estructura, un calentamiento de 10 min con actividades introductorias de cada uno de los deportes; una parte principal de $35 \mathrm{~min}$ donde se desarrollan contenidos técnico-tácticos de cada una de las especialidades por medio de circuitos; una parte final de 12 min que consistió en juegos relacionados con los contenidos; y una reunión final de tres minutos de duración. Se establecieron cinco zonas de intensidad siguiendo las recomendaciones 
valores relativos entre el pre y post-test (tabla 1, 2, 3). Esto pone de manifiesto que doce semanas de actividad física pueden tener una repercusión directa a nivel antropométrico, que si bien, solo se manifiesta en el peso, puede que, con un programa más prolongado en el tiempo, esto fuese extensible a otras variables. Dado, además, que las variables antropométricas están muy condicionadas por el volumen de actividad física que se realice a lo largo de la semana (Torres-Luque et al., 2014), podría ser interesante seguir profundizando en esta línea con la determinación del porcentaje graso (Torres-Luque, Calahorro-Cañada, LaraSánchez, Garatachea \& Nikolaidis, 2015).

A nivel de condición física, especialmente sobre la flexibilidad, aparece un descenso estadísticamente significativo tras el programa de intervención en el grupo total $(\mathrm{p}<0,05)$. Sin embargo, en los resultados diferenciados por géneros (tabla 2 y 3 ) a pesar de existir un descenso de la flexibilidad isquiosural, es estadísticamente significativa en el género femenino $(p<0,01)$. Resultados muy diferentes a los registrados por Ho et al., (2017), donde niños y niñas de 12 años mejoraban su flexibilidad isquiosural tras una intervención de 8 semanas de actividades deportivas. Es cierto a su vez, que, en estudios previos, la tendencia es a mostrar resultados de flexibilidad mayores en chicas que en chicos (De Miguel-Etayo et al., 2014; Roriz et al., 2014; Torres-Luque et al., 2014). Estos resultados, se pueden deber a que la estructura de las sesiones desarrolladas en este trabajo carece de una parte de estiramientos específica, tan recomendados en etapas de formación, especialmente entre los 5 y 12 años (Lloyd, Oliver, Meyers, Moody \& Stone, 2012). No se debe olvidar que la pérdida de flexibilidad isquiosural está asociadas a un elevado riesgo de dolor lumbaru otras patologías en jóvenes deportistas (Sadler, Spink, Ho, Jonge \& Chuter, 2017). De hecho, aparecen referencias sobre la importancia de la flexibilidad isquisural en el deporte para la prevención de lesiones y la optimización del rendimiento deportivo (Wan, Qu, Garret, Liu \& Yu, 2017; Van Der Horst, Priesterbach, Backx \& Smits, 2017; Silder, Thelen \& Heiderscheit, 2010), por lo tanto, en futuras intervenciones sería necesario considerar incluir un bloque de estiramientos específicos que ayuden a paliar estos resultados.

En cuanto a la capacidad de fuerza, se produce un incremento estadísticamente significativo en la fuerza del tren inferior (test de salto horizontal) $(\mathrm{p}<0,001)$, no siendo destacable en la fuerza de prensión manual o la velocidad con cambios de dirección (test de 4x10m). Los datos que se muestran, están dentro de la normalidad para sujetos con normopeso (Campos Jara et al., 2016) e incluso, para poblaciones que hacen cinco o seis horas de actividad física a la semana (Torres-Luque et al., 2014), si bien es cierto que las mejoras se dan solo y exclusivamente en el salto horizontal tanto en el grupo total como por género (tabla 1, 2, 3). Esto tiene una reflexión importante, ya que el salto horizontal partía con valores que ya estaban clasificados como bajos (Ruiz et al., 2011) y en estudiantes chilenos los valores posteriores estarían dentro de la normalidad para sujetos con bajo-peso (Campos Jara et al., 2016). Con lo que el programa sería efectivo, donde existe un ligero descenso de la masa corporal y un incremento acentuado de la fuerza de miembros inferiores, donde los porcentajes de mejora están en torno al 10\%. Löfgren et al (2013) ya indicaban que el entrenamiento de fuerza en el contexto escolar colabora a aumentar la fuerza muscular. A su vez, este test está también condicionado por la coordinación dinámica general, ya que se puede utilizar el balanceo de los brazos (Ruiz et al., 2011; Ortega et al., 2011), lo que implica la importancia de esta cualidad presente en el deporte base, donde a pesar de que los sujetos estaban familiarizados con el test, el hecho de realizar deportes individuales o colectivos puede colaborar a su incremento. Importante a su vez, destacar que el salto horizontal se trata de una de las habilidades motrices básicas altamente relacionada con las habilidades motrices específicas de desplazamiento de los deportes desarrollados en el programa de intervención: taekwondo, atletismo, patinaje, hockey, balonmano y baloncesto (Lubans, Morgan, Cliff \& Okely, 2010).

Sin embargo, al controlarse la estructura y orientación de la sesión en cada caso, pero no el contenido o metodología de enseñanza, parece ser que el desarrollo de aspectos técnico-tácticos de las citadas modalidades haya derivado en unos efectos más liviano sobre la velocidad, y que esto conlleve menores cambios en el test de $4 \times 10 \mathrm{~m}$. Son diversos los estudios que indican que los chicos tienen mejores resultados que las chicas en pruebas de velocidad (De Miguel-Etayo et al., 2014; LópezGallego, Lara-Sánchez, Espejo \& Cachón, 2016). Además, Torres-Luque et al. (2014) afirman dos cuestiones importantes, por un lado, que según se incrementa el volumen de práctica de actividad física semanal, los valores en velocidad tienen a reducirse; $\mathrm{y}$ por otro, que, independientemente de esta circunstancia, los chicos tienen mejores resultados de velocidad que las chicas. Esto indica que es necesario un mayor control de los programas de actividad física extraescolares que se generan en Chile para población sana.

A pesar de los pros y contras del programa de actividad física en cuanto a estas variables, destaca fundamentalmente los parámetros cardiorrespiratorios. El consumo máximo de oxígeno obtiene valores muy bajos en el inicio del programa (tabla 1). Los mismos, teniendo en cuenta criterios de sexo y edad (Adegboye et al., 2011), serían datos de un consumo de oxígeno no saludable (Calahorro-Cañada et al., 2015), aspecto que debe de preocupar, porque ha sido demostrado que este parámetro es un indicador directo de salud en niños y adolescentes (Ortega et al., 2008). Es cierto que estos datos iniciales son similares a otros estudios en estudiantes chilenos (Campos Jara et al., 2016), pero que distan de datos en europeos (Ortega et al., 2008; Arriscado, Muros, Zabala \& Dalmau, 2015). Sin embargo, es destacable y una de las fortalezas de este trabajo, que doce semanas de un programa estructurado de actividad física tres veces por semana, conlleva un incremento estadísticamente significativo tanto en niños como en niñas $(p<0,01)$ (tablas 2,3). El incremento, en valores relativos está en torno a un 7\% en tan solo doce semanas, que, aunque se conoce que es suficiente para mejoras en este sentido en población adolescente (Shatilin et al., 2009; Carrasco et al., 2014), es un hecho destacable. De hecho, se sabe que estudiantes con un consumo de oxígeno no saludable, obtienen mayor nivel de actividad física los días que hay clases de educación física, a lo cual, si se le incrementa con actividades extraescolares los resultados pueden acelerarse (Calahorro-Cañada, Torres Luque, López Fernández \& Carnero 2017). No obstante, si llama la atención la diferencia existente entre el género masculino y femenino. Aunque ambos grupos mejoren, los resultados de las chicas son más llamativos. Como premisa, los sujetos de género masculino, tienden a tener mayor consumo de oxígeno que el género femenino (De Miguel-Etayo et al., 2014; López-Gallego, Lara-Sánchez, Espejo \& Cachón, 2016). Esto puede deberse a que los componentes de las sesiones de entrenamiento dentro del programa de actividad física del presente estudio estaban compuestos por jóvenes de ambos sexos. A su vez, en cada parte de la estructura de la sesión se trabajaba dentro de una de las zonas de intensidad previamente establecidas individualmente (ACSM, 2014). Dado, que es un intervalo de intensidad, es posible que la misma haya podido influir de manera diferente en el género masculino como en el femenino y de ahí estos resultados. La necesidad de ser más exhaustivos en el control de las cargas de entrenamiento en población joven es indiscutible (SánchezSánchez et al., 2015), por lo que hay que seguir generando evidencias que ayuden a crear conciencia de la necesidad de dar un paso más allá en el control de estos programas extraescolares, algo necesario en un país como Chile.

Un parámetro asociado es la frecuencia cardiaca submáxima, donde en el caso de los estudiantes chilenos, es determinada por medio del test de CAFRA, prueba específica de este país (MINEDUC, 2011). Aunque la mejora antes y después del programa, solo es estadisticamente significativa cuando el test se realiza a $8 \mathrm{~km} / \mathrm{h}(\mathrm{p}<0,05)$, las mejoras son patentes a las tres velocidades submáximas contempladas, en torno al 27\% (tabla 2, 3). La eficiencia cardiaca después de un programa de actividad fisica, es algo altamente contrastado. En este caso, el test marca valores de frecuencia cardiaca preocupantes, cuando se exceden los 160 lat/min a la velocidad de $6 \mathrm{~km} / \mathrm{h}$ (Agencia de calidad de la educacion, 2013), lo que no ocurre hasta la velocidad de $7-8 \mathrm{~km} / \mathrm{h}$. Es cierto, que una de las limitaciones de este estudio es que no se ha evaluado el nivel de actividad fisica del programa y tampoco el tiempo útil de las sesiones, a pesar de ser estructurado en todos los casos, que 
podrian ser datos interesantes para observar la efectividad del programa. Son diversos los autores que marcan como un programa de actividad física multi-deporte tiene beneficios en la salud en general (GarcíaHermoso et al., 2016) y que los mismos deben estar bien estructurados y dirigidos. Este estudio pone de manifiesto que, aunque en muchos lugares y poblaciones, no se tenga un instrumental caro y sofisticado que permita generar un seguimiento y control de un programa de actividad física de una manera más exhaustiva, no debe ser un limitante para llevarlo a cabo, ya que existen mejoras que, de ser más continuadas en el tiempo, puedan establecerse como mejoras que impliquen un hábito saludable y que perduren hasta la edad adulta (Ortega et al., 2005).

Sobre el equilibrio dinámico a través del TBY, los sujetos de este estudio muestran resultados similares a los registrados por Linek, Sikora, Wolny y Saulicz (2017) en jugadores de futbol de 15 años, en la dirección frontal anterior. Sin embargo, los valores obtenidos en las direcciones posteriores (plano medio y plano lateral), los jugadores de futbol poseen valores entre 106 y $111 \mathrm{~cm}$ de media, muy superiores a los encontrados en los jóvenes de este estudio. No obstante, parece ser que los efectos de este programa de intervención han provocado una mejora significativa en el equilibrio dinámico de los sujetos, y los datos alcanzados en ambos géneros en las direcciones de plano medio y lateral (85 y $91 \mathrm{~cm}$ ) tras la fase de intervención en el presente trabajo, son más similares a los mostrados por Alhusaini, Alnahdi, Melan, Aldali, AlMutairi y Alenzi (2017) que muestra valores entre 99 y 104 cm. Por otro lado, los estudios revisados muestran valores superiores en el plano lateral que en el plano medial (Linek et al., 2017; Alhusaini et al., 2017; Overmoyer y Reiser, 2015) similar a datos obtenidos en el presente estudio. Esto se debe, principalmente a que los factores clave en este test: la movilidad de la articulación del miembro inferior, especialmente de rotadores externos y abductores; y el rendimiento de la fuerza de los extensores de la articulación de la rodilla (Walaszek, Chwala, Walaszek, Burdacki \& Blaszczuk, 2017). Teniendo en cuenta el carácter multidireccional que poseen los deportes practicados en la intervención de este estudio (taekwondo, atletismo, patinaje, hockey, balonmano y baloncesto), parece lógica la mejora en el equilibrio dinámico en los sujetos valorados.

Este trabajo no está exento de limitaciones, entre las que destaca algunas de las ya indicadas. El programa de actividad física generado es el que realizan multitud de centros escolares en Santiago de Chile, en población sana. Esto implica que el acceso a material e instrumental sea limitado, por lo que fundamentalmente en el control de las intensidades de las sesiones, el empleo de pulsometros, escalas de percepción subjetiva de esfuerzo, etc. debería ser algo que se implementase de manera rutinaria. Dados los resultados, quizás la confección de los grupos con un carácter mixto respecto al género, haya podido influir en alguna variable. Como fortaleza, aunque se hay destacado previamente, es un estudio encuadrado en lo que actualmente son actividades extraescolares en chicos y chicas de estas edades que no realizan ninguna actividad física extraescolar, y donde, por medio de este programa se puede demostrar que en poco tiempo se pueden conseguir mejoras. Función futura será analizar efectos más longitudinales y control de la adherencia al programa de actividad física que aquí no se han determinado.

\section{Conclusión}

Un programa de actividades físicas extraescolares de 12 semanas de duración en jóvenes sanos y chilenos de entre 13 y 14 años obtiene beneficios a nivel cardiovascular, salto de longitud y equilibrio dinámico; no obteniendo mejoras significativas en parámetros relacionados con la fuerza o velocidad.

Sepone de manifiesto la necesidad de la evaluación de programas de actividad física extraescolar en jóvenes sanos con el objetivo de mejorar intervenciones futuras.

\section{Referencias}

Adegboye, A. R., Anderssen, S. A., Froberg, K., Sardinha, L. B.,
Heitmann, B. L., Steene-Johannessen, J., ... \& Andersen, L. B. (2011). Recommended aerobic fitness level for metabolic health in children and adolescents: a study of diagnostic accuracy. British Journal of Sports Medicine, 45(9), 722-728.

Agencia de Calidad de la Educación (2013). Informe de Resultados Estudio Nacional de Educación Física 2013. Ministerio de Educación.

Alhusaini, A. A., Alnahdi, A. H., Melam, G., Aldali, A. Z., Al-Mutairi, M. S., \& Alenzi, A. R. (2017). Normative values of Y Balance Test and isometric muscle strength among Saudi school children. Physikalische Medizin, Rehabilitationsmedizin, Kurortmedizin, 27(03), 164-170.

American College of Sports Medicine; Pescatello, L.S., Arena, R., Riebe, D., Thompson, P.D., eds. (2014). ACSM's Guidelines for Exercise Testing and Prescription. 9th ed.; Philadelphia (PA): Wolters Kluwer/Lippincott Williams \& Wilkins Health.

Arriscado, D., Muros, J. J., Zabala, M., \& Dalmau, J. M. (2015). Physical activity habits in schoolchildren: influential factors and relationships with physical fitness. Nutrición hospitalaria, 31(3), 1232-1239.

Bailey, R. (2006). Physical education and sport in schools: A review of benefits and outcomes. Journal of school health, 76(8), 397-401.

Brazendale, K., Beets, M. W., Weaver, R. G., Chandler, J. L., Randel, A. B., Turner-McGrievy, G. M., ... \& Ward, D. S. (2017). Children's Moderate to Vigorous Physical Activity Attending Summer Day Camps. American Journal of Preventive Medicine, 53(1), 78-84.

Burrows, R., Díaz, E., Sciaraffia, V., Gattas, V., Montoya, A., \& Lera, L. (2008). Hábitos de ingesta y actividad física en escolares, según tipo de establecimiento al que asisten. Revista médica de Chile, 136(1), 53-63.

Calahorro-Cañada, F., Torres-Luque, G, López-Fernández, I., \& Carnero, E. A. (2015). Análisis fraccionado de la actividad física desarrollada en escolares. Revista de Psicología del Deporte, 24(2).

Calahorro Cañada, F., Torres Luque, G., López Fernández, I., \& Carnero, E. A. (2017). Is physical education an effective way to increase physical activity in children with lower cardiorespiratory fitness? Scandinavian journal of medicine \& science in sports, 27(11), 1417-1422.

Campos Jara, C., Delgado Floody, P., Caamaño Navarrete, F., Guzmán Guzmán, I., Cresp Barría, M., Jerez Mayorga, D., ... \& Osorio Poblete, A. (2016). Alteraciones en el rendimiento físico de escolares: los Test Cafra y Navette y su asociación con la obesidad y riesgo cardiometabólico. Nutrición Hospitalaria, 33(4), 808-813.

Carrasco Fernández, J. C., Lara-Sánchez, A. J., \& Torres-Luque, G. (2014). Efectos de un programa de entrenamiento de fútbol sobre la condición física en jugadores jóvenes. Kronos, 13(1).

Casterad, Z., \& Ostariz, S. (2005). Dimensiones De La Condición Física Saludable: Evolución Según Edad y Género. Medicina, 5, 50-67.

Cladellas, R., Muntada, MC., Badia, M., Gotzens, C. (2013). Actividades extraescolares y rendimiento académico en alumnos de primaria. European Journal of investigation in Health, Psychology and Education, 3(2), 87-97

Cladellas, R., Muntada, MC., Gotzens, C., Badia, M., Dezcallar, T. (2015). Patrones de descanso, actividades físico-deportivas extraescolares y rendimiento académico en niños y niñas de primaria. Revista de Psicología del Deporte, 24(1), 53-59.

Cole, T. J., Bellizzi, M. C., Flegal, K. M., \& Dietz, W. H. (2000). Establishing a standard definition for child overweight and obesity worldwide: international survey. Bmj, 320(7244), 1240.

De Miguel-Etayo, P., Gracia-Marco, L., Ortega, F. B., Intemann, T., Foraita, R., Lissner, L., Oja, L., Barba, G., Michels, N., Tornaritis, M., Molnár, D., Pitsiladis, Y., Ahrens, W. \& Moreno, L. A. (2014). Physical fitness reference standards in European children: the IDEFICS study. International Journal of Obesity, 38, S57-S66.

García-Hermoso, A., Carmona-López, M. I., Saavedra, J. M., \& Escalante, Y. (2014). Ejercicio físico, desentrenamiento y perfil lipidico en niños obesos: una revisión sistemática. Archivos ar- 
gentinos de pediatría, 112(6), 519-525.

Godard, C., Román, M., Rodríguez, M. D. P., Leyton, B., \& Salazar, G. (2012). Variabilidad de la actividad física en niños chilenos de 4 a 10 años: estudio por acelerometría. Archivos argentinos de pediatría, 110(5), 388-393.

Ho, F. K. W., Louie, L. H. T., Wong, W. H. S., Chan, K. L., Tiwari, A., Chow, C. B., ... \& Cheung, Y. F. (2017). A sports-based youth development program, teen mental health, and physical fitness: an RCT. Pediatrics, e20171543.

Kushner, R. F. (2014). Weight loss strategies for treatment of obesity. Progress in Cardiovascular Diseases, 56(4), 465-472.

Linek, P., Sikora, D., Wolny, T., \& Saulicz, E. (2017). Reliability and number of trials of $\mathrm{Y}$ Balance Test in adolescent athletes. Musculoskeletal Science and Practice, 31: 72-75.

Lloyd, R. S., Oliver, J. L., Meyers, R. W., Moody, J. A., \& Stone, M. H. (2012). Long-term athletic development and its application to youth weightlifting. Strength \& Conditioning Journal, 34(4), 55-66.

Löfgren, B., Daly, R.M., Nilsson, J.A., Dencker, M. \& Karlsson, M.K. (2013). An increase in school-based physical education increases muscle strength in children. Medicine and Science in Sports and Exercise, 45(5), 997-1003.

López-Gallego, F.J., Lara-Sánchez, A.J., Espejo, N. \& Cachón, J. (2016). Influencia del género, la edad y el nivel de actividad física en la condición física de alumnos de educación primaria. Revisión Bibliográfica. Retos. Nuevas tendencias en educación física, deporte y recreación, 29, 129-133.

Lubans, D. R., Morgan, P. J., Cliff, D. P., Barnett, L. M., \& Okely, A. D. (2010). Fundamental movement skills in children and adolescents. Sports medicine, 40(12), 1019-1035.

Mahoney, J.L., Cairns, B.D. y Farmer, T.W. (2003). Promoting interpersonal competence and educational success through extracurricular activity participation. Journal of Educational Psychology, 95, 409-418.

Martínez-Gómez, D., Eisenmann, J. C., Gómez-Martínez, S., Veses, A., Marcos, A., \& Veiga, O. L. (2010). Sedentarismo, adiposidad y factores de riesgo cardiovascular en adolescentes. EstudioAFINOS. Revista española de cardiología, 63(3), 277-285.

Martínez-Vizcaíno, V., Sánchez-López, M., Notario-Pacheco, B., SalcedoAguilar, F., Solera-Martínez, M., Franquelo-Morales, P., ... \& MoraRodríguez, R. (2014). Gender differences on effectiveness of a schoolbased physical activity intervention for reducing cardiometabolic risk: a cluster randomized trial. International Journal of Behavioral Nutrition and Physical Activity, 11(1), 154.

MINEDUC (2011). Informe de resultados en Educación Física. SIMCE. Ministerio de Educación. Unidad de Curriculum y evaluación. Santiago. MINEDUC.

Ministerio de Salud (2011). Estrategia nacional de salud, para el cumplimiento de los objetivos sanitarios de la década 2011-2020. Extraído de: http://web.minsal.cl/portal/url/item/ c4034eddbc96ca6de0400101640159b8. pdf.

Molinero, O., Castro-Piñero, J., Ruiz, J.R., González Montesinos, J.L., Mora, J., \& Márquez, S. (2010). Conductas de salud en escolares de la provincia de Cádiz, Nutrición Hospitalaria, 25(2), 280-289.

Ortega, F. B., Artero, E. G., Ruiz, J. R., España-Romero, V., JiménezPavón, D., Vicente-Rodríguez, G., ... \& Ciarapica, D. (2011). Physical fitness levels among European adolescents: the HELENAstudy. British journal of sports medicine, 45(1), 20-29

Ortega, F. B., Ruiz, J. R., Castillo, M. J., Moreno, L. A., González-Gross, M., Warnberg, J. y Gutiérrez, A. (2005). Bajo nivel de forma física en los adolescentes españoles. Importancia para la salud cardiovascular futura (Estudio AVENA). Revista Española de Cardiología, 58, 898909.

Ortega, F., Ruiz, J., Castillo, M. \& Sjöström M. (2008). Physical fitness in childhood and adolescence: a powerful marker of health. International Journal of Obesity, 32, 1-11.

Overmoyer, G. V., \& Reiser, R. F. (2015). Relationships Between LowerExtremity Flexibility, Asymmetries, and the Y Balance Test. The Journal of Strength \& Conditioning Research, 29(5), 1240-1247.

Plisky, P. J., Gorman, P. P., Butler, R. J., Kiesel, K. B., Underwood, F. B., \& Elkins, B. (2009). The reliability of an instrumented device for measuring components of the star excursion balance test. North American journal of sports physical therapy: NAJSPT, 4(2), 92.

Roriz, M.S., Seabra, A., Freitas, D., Eisenmann, J. C. \& Maia J. (2014). Physical fitness percentile charts for children aged 6-10 from Portugal. The Journal of Sports Medicine and Physical Fitness, 54(6), 780-92.

Ruiz, J. R., España Romero, V., Castro Piñero, J., Artero, E. G., Ortega, F. B., Cuenca García, M., ... \& Gutiérrez, A. (2011). Batería ALPHAFitness: test de campo para la evaluación de la condición física relacionada con la salud en niños y adolescentes. Nutrición Hospitalaria, 26(6), 1210-1214.

Sadler, S. G., Spink, M. J., Ho, A., De Jonge, X. J., \& Chuter, V. H. (2017). Restriction in lateral bending range of motion, lumbar lordosis, and hamstring flexibility predicts the development of low back pain: a systematic review of prospective cohort studies. BMC musculoskeletal disorders, 18(1), 179.

Saìnchez-Saìnchez, J., Peìrez, S., Yague, J.M., Royo, J.M. \& Martiìn, J.L. (2015). Aplicacioìn de un programa de entrenamiento de fuerza en futbolistas joìvenes. Revista Internacional de Medicina y Ciencias de la Actividad Física y el Deporte, 15(57), 45-59.

Shalitin, S., Ashkenazi-Hoffnung, L., Yackobovitch-Gavan, M., Nagelberg, N., Karni, Y., Hershkovitz, E., ... \& Phillip, M. (2009). Effects of a twelve-week randomized intervention of exercise and/or diet on weight loss and weight maintenance, and other metabolic parameters in obese preadolescent children. Hormone Research in Paediatrics, 72(5), 287-301.

Silder, A., Thelen, D. G., \& Heiderscheit, B. C. (2010). Effects of prior hamstring strain injury on strength, flexibility, and running mechanics. Clinical Biomechanics, 25(7), 681-686.

Torres-Luque, G, Calahorro-Cañada, F., Lara-Sánchez, A., Garatachea, N., Nikolaidis, P.T. (2015) Body composition using bioelectrical impedance analysis in elite young soccer players: the effects of age and playing position. Sport Science for Health ,11, 203-210.

Torres-Luque, G., Carpio, E., Lara Sánchez, A., \& Zagalaz Sánchez, M. (2014). Niveles de condición física de escolares de educación primaria en relación a su nivel de actividad física y al género. Retos. Nuevas tendencias en educación física, deporte y recreación, (25), 17-22.

Valdés, P. \& Yancin, J. (2016) Análisis de la condición física, tipo de actividad física realizada y rendimiento académico en estudiantes de educación secundaria. Retos. Nuevas tendencias en educación física, deporte y recreación, 30, 64-69.

Van Der Horst, N., Priesterbach, A., Backx, F., \& Smits, D. W. (2017). Hamstring-and-lower-back flexibility in male amateur soccer players. Clinical journal of sport medicine, 27(1), 20-25.

Venturelli, M., Trentin, F., Bucci, M. (2007). Strength training for young soccer players. Journal of Sports Science and Medicine, Suppl. 10, 84.

Villa-Vicente, G., Sánchez-Collado, P., Rodríguez-Marroyo, JA., MartínezCastañeda, R., Ávila-Ordás, M.C., Muñoz- Weigand, C. (2007). La escasa actividad física y deportiva escolar genera, más que la alimentación, sobrepeso, obesidad infantil y riesgo de síndrome metabólico. Premio de Investigación en Medicina del Deporte. Universidad de Oviedo.

Walaszek, R., Chwa³a, W., Walaszek, K., Burdacki, M., \& B³aszczuk, J. (2017). Evaluation of the accuracy of the postural stability measurement with the Y-Balance Test based on the levels of the biomechanical parameters. Acta of bioengineering and biomechanics, 19(2).

Wan, X., Qu, F., Garrett, W. E., Liu, H., \& Yu, B. (2016). Relationships among hamstring muscle optimal length and hamstring flexibility and strength. Journal of Sport and Health Science, 6(3): 275-282

Wang, Y., \& Lim, H. (2012). The global childhood obesity epidemic and the association between socio-economic status and childhood obesity. International Review Psychiatry, 24(3), 176-188.

Waters, E., de Silva Sanigorski, A., Burford, B. J., Brown, T., Campbell, K. J., Gao, Y., ... \& Summerbell, C. D. (2011). Interventions for preventing obesity in children. The Cochrane Library, 12.

WHO (2009). Population-based prevention strategies for childhood obesity. Report of a WHO forum and technical meeting. Ginebra: Publications of the World Health Organization. 